\title{
Effects of Povidone-Iodine or Ethanol Exposure on Bone Formation at the Osteotomy Site of the Proximal Tibia in Rats
}

\author{
Itsuki Nagahata $\mathbb{C}^{\circ}$, Naohisa Miyakoshi* ${ }^{\circledR}$, Yuji Kasukawa ${ }^{\circledR}$, Hiroyuki Tsuchie ${ }^{\circledR}$, \\ Yusuke Yuasa ${ }^{\circledR}$, Manabu Akagawa ${ }^{(0)}$, Yuichi Ono $\left.{ }^{(}\right)$, Chiaki Sato ${ }^{\mathbb{D}}$, Koji Nozaka ${ }^{(}$, \\ Michio Hongo $\mathbb{D}^{\circ}$, Yoichi Shimada $\mathbb{}$
}

Department of Orthopedic Surgery, Akita University Graduate School of Medicine, Akita, Japan

Email: nagahata.itsuki.1986@gmail.com, ^miyakosh@doc.med.akita-u.ac.jp, kasukawa@doc.med.akita-u.ac.jp, tuchikiti@yahoo.co.jp,yuasayusuke@gmail.com, prcmcn3@gmail.com,yuichi.ono24@gmail.com, chiaki.sato@med.akita-u.ac.jp,nozakak@med.akita-u.ac.jp,mhongo@doc.med.akita-u.ac.jp, seikei@doc.med.akita-u.ac.jp

How to cite this paper: Nagahata, I., Miyakoshi, N., Kasukawa, Y., Tsuchie, H., Yuasa, Y., Akagawa, M., Ono, Y., Sato, C., Nozaka, K., Hongo, M. and Shimada, Y. (2019) Effects of Povidone-Iodine or Ethanol Exposure on Bone Formation at the Osteotomy Site of the Proximal Tibia in Rats. Open Journal of Orthopedics, 9, 265-272. https://doi.org/10.4236/ojo.2019.912027

Received: October 13, 2019

Accepted: December 16, 2019

Published: December 19, 2019

Copyright $\odot 2019$ by author(s) and Scientific Research Publishing Inc. This work is licensed under the Creative Commons Attribution International License (CC BY 4.0).

http://creativecommons.org/licenses/by/4.0/ c) (i) Open Access

\begin{abstract}
Background: Povidone-iodine (PVI) irrigation is currently used to decrease the frequency of postoperative surgical site infections. Ethanol (EtOH) is sometimes applied to prevent local recurrence after curettage of benign bone tumors. However, the effects of PVI and $\mathrm{EtOH}$ on surrounding soft tissue and on bone union are unclear. The purpose of this study was to determine whether PVI or EtOH adversely affects the cancellous bone healing of the osteotomy site at the proximal tibia in rats. Methods: A cancellous bone osteotomy was performed at the right proximal tibia in 4-month-old, female, Sprague Dawley rats. Vehicle, 10\% PVI, or 95\% EtOH-soaked gauze was inserted into the osteotomy site and maintained for 6 minutes. The rats were euthanized 2 or 4 weeks after the osteotomy. Results: Two weeks after treatment, the bone union rate was significantly higher in the vehicle group than in the PVI group and the EtOH group $(\mathrm{p}<0.001)$. However, the bone union rate was not significantly different between the PVI and EtOH groups. There was no significant difference among the three groups in the bone union rate 4 weeks after treatment. Conclusion: PVI or EtOH delayed bone union of the cancellous bone osteotomy site of the proximal tibia in the early phase ( 2 weeks), but not at 4 weeks, in rats.
\end{abstract}

\section{Keywords}

Cancellous Bone Union, Povidone-Iodine, Ethanol 


\section{Introduction}

In the field of orthopedic surgery, a surgical site infection is a very serious clinical problem following surgery for severe trauma with large bone defects and damage to soft tissues. To reduce the risk of surgical site infection, many surgeons are using antimicrobial wound irrigation [1]. Povidone-iodine (PVI) irrigation of the surgical field is currently used in urologic, cardiovascular, and orthopedic surgery, since it has been shown to decrease the frequency of postoperative surgical site infections [2] [3]. Although a diluted 1\% PVI solution is safe to use on wounds to reduce infection in children with appendicitis [4] and in spinal surgery [5], PVI might have a rapid and detrimental effect on human osteoblast proliferation [6]. However, the effects of PVI on bone healing, especially cancellous bone healing or bone bonding, have not yet been fully elucidated.

On the other hand, an additional procedure is sometimes required during surgery for bone tumor resection. For example, although a giant cell tumor is a common benign primary bone tumor, it has a locally aggressive recurrence rate of $30 \%-50 \%$ after simple curettage [7]. For that reason, various local adjuvant therapies are being used in addition to curettage to reduce the recurrence rate. Application of ethanol (EtOH) is one of the adjuvant therapies that reduces the recurrence rate to $10 \%-16 \%$ [8]. Application of $95 \%$ EtOH causes tumor necrosis and few EtOH-related complications [9]. However, the effects of EtOH on surrounding soft tissue, bone union, and neurovascular structures are unclear.

In the present study, whether PVI or EtOH adversely affects cancellous bone healing of the osteotomy site at the proximal tibia in rats was examined.

\section{Materials and Methods}

\section{Animals}

Four-month-old, female, Sprague Dawley rats (Charles River Laboratory Inc., Kanagawa, Japan) were used in this study and housed in a controlled environment at $22^{\circ} \mathrm{C}$ with a 12 -h light/dark cycle. The rats were allowed free access to water and pair-fed standard food (CE-2; Clea Japan Inc., Tokyo, Japan) containing $1.14 \%$ calcium, $1.06 \%$ phosphorus, and $250 \mathrm{IU}$ vitamin D3 per $100 \mathrm{~g}$.

\section{Experimental Protocol and Surgical Procedure}

All animal experiments conformed to the "Guidelines for Animal Experimentation" of Akita University School of Medicine.

A cancellous bone osteotomy was performed at the right proximal tibia in 4 -month-old, Sprague Dawley, female rats $(\mathrm{n}=42)$. A median parapatellar incision from the knee joint through the ankle joint was made at the right hind limb, and complete mid-sagittal osteotomy from the joint surface to the tibial diaphysis was performed using an electric bone saw with the animals under general anesthesia with ketamine (Sankyo, Tokyo, Japan) and xylazine (Zenoaq, Fukushima, Japan), as previously reported [10] (Figure 1(a)). Normal saline as a vehicle, 10\% PVI, or 95\% EtOH-soaked gauze was inserted into the osteotomy site at the proximal tibia and maintained for 6 minutes, based on a previous report 
[8] [11] (Figure 1(b)). After removing the gauze, the osteotomy site was then circled with a circumferential wire suture (diameter $0.4 \mathrm{~mm}$ ) [12] [13] (Figure $1(\mathrm{c})$ ). After surgery, the animals were allowed to move freely in their cages. No animal had an abnormal gait or impaired locomotion postoperatively.

The rats were divided into the following three groups: 1 ) vehicle group ( $\mathrm{n}=$ $14) ; 2)$ PVI group ( $n=14)$; and 3) EtOH group $(n=14)$. The rats were euthanized 2 or 4 weeks after the osteotomy under anesthesia with pentobarbital (Nembutal, Abbott Laboratories, Chicago, IL). The right tibia was harvested and fixed in $70 \%$ alcohol until preparation for histological examination (Figure 2).

\section{Bone Histomorphometry}

The right proximal halves of the tibiae, including the osteotomy sites, were decalcified with neutral $10 \%$ ethylene diamine tetra-acetic acid for 3 weeks and embedded in paraffin. Five serial $3-\mu \mathrm{m}$-thick mid-frontal sections were prepared for hematoxylin and eosin staining for cancellous bone histomorphometry.

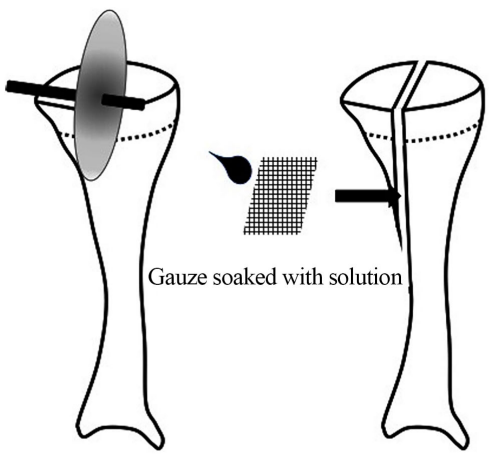

(a) (b)

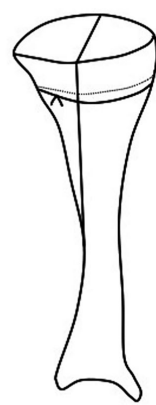

(c)

Figure 1. Bone osteotomy procedure. Schema of cancellous bone osteotomy. Complete mid-sagittal osteotomy from the knee joint surface as far as possible is performed using an electrically powered bone saw (a); the osteotomized proximal tibia is opened bilaterally, and gauze soaked with each solution is inserted for 6 minutes (b); the gauze is removed, and then the osteotomy site is fixed with cerclage wiring (diameter $0.4 \mathrm{~mm}$ ) circumferentially (c).

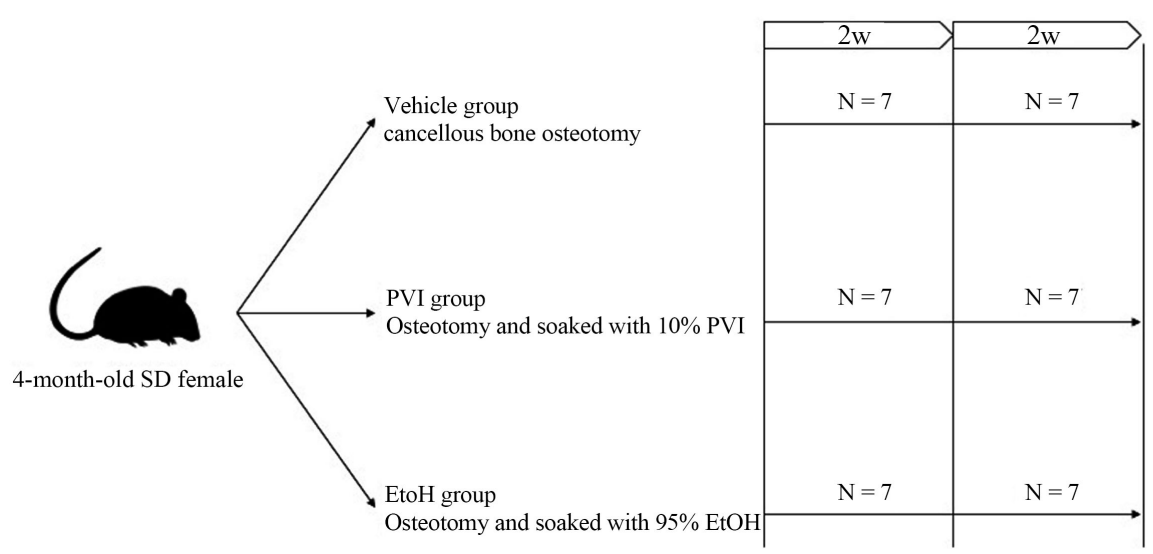

Figure 2. Schema of the experimental protocol. Four-month-old, Sprague Dawley rats are assigned to three groups (vehicle, PVI, or EtOH groups) and euthanized at 2 or 4 weeks. 
A semiautomatic graphic system (Histometry RT Camera, System Supply Co., Nagano, Japan), at 100x magnification, was used to measure the length of bone union, defined as bone-to-bone bonding at the osteotomy line (Figure 3); the measurement range was from $400 \mu \mathrm{m}$ to $1000 \mu \mathrm{m}$ distal from the growth plate [14] (Figure 3, yellow area). Cartilaginous bonding was also defined as bony union, whereas fibrous bonding was regarded as nonunion. The total length of bone union including bone-to-bone bonding and cartilaginous bonding and the length of non-union with fibrous bonding at the center of the osteotomy line were measured. Then, the proportion of bone union in the total length of the osteotomy line was calculated [15].

\section{Statistical Analyses}

All values are expressed as means \pm standard deviation. One-way analysis of variance (ANOVA) was performed to evaluate the effects of PVI and EtOH. Differences among groups at each time point were evaluated using the Bonferroni test for multiple comparisons with ANOVA. All statistical analyses were performed with EZR (Easy R) statistical software (The R Foundation for Statistical Computing, Vienna, Austria, version 3.4.3) [16]. Values of $\mathrm{p}<0.05$ were considered significant.

\section{Results}

\section{Histological Findings of Cancellous Bone Union at the Osteotomy Site (Figure} 4)

Figure 4 shows the histological findings of the cancellous bone union at the osteotomy site of the proximal tibia at 2 weeks. There was some fibrous tissue at the osteotomy site in the PVI (Figure 4(b)) and EtOH groups (Figure 4(c)). Fibrous tissue at the osteotomy site was defined as non-union. Thus, much more cancellous bone union was observed in the vehicle group (Figure 4(a)) than in the PVI (Figure 4(b)) and EtOH groups (Figure 4(c)) at 2 weeks.

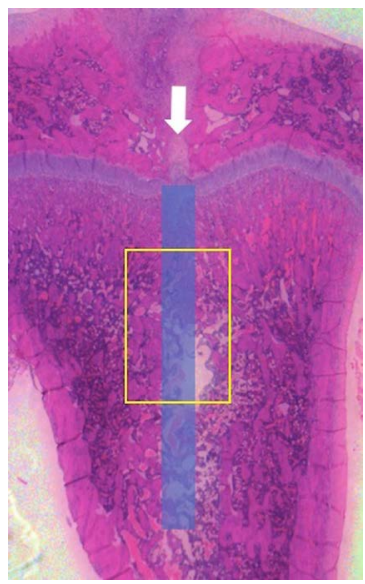

Figure 3. Measurement range of bone histomorphometry. Histological section stained with hematoxylin and eosin at the proximal tibia with the osteotomy site (20x). The white arrow and the blue area show the osteotomy site. The area enclosed by the yellow frame is the measurement range from $400 \mu \mathrm{m}$ to $1000 \mu \mathrm{m}$ from the growth plate. 


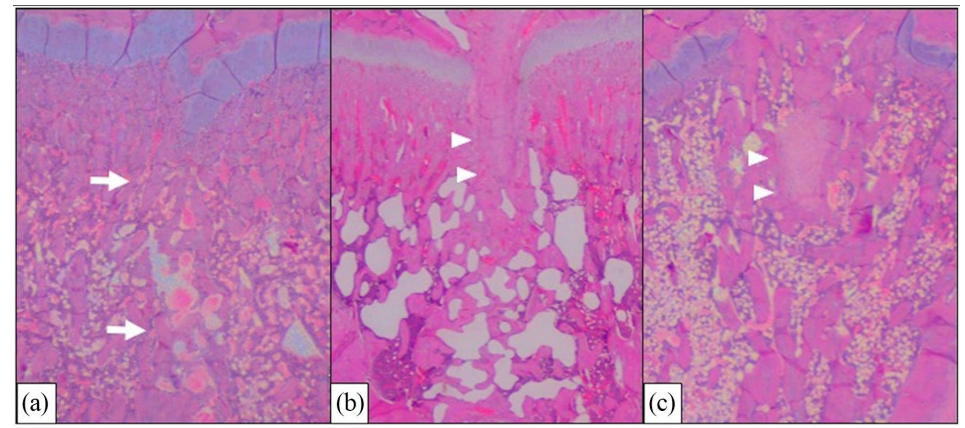

Figure 4. Histological sections of the osteotomy site at 2 weeks, stained with hematoxylin and eosin at 20x magnification. At 2 weeks, the osteotomy site of the vehicle group (a) is filled with cancellous bone (arrows) and fibrous bone. On the other hand, the PVI group (b) and the EtOH group (c) are filled with more fibrous bone (arrow heads) than the vehicle group.

\section{Bone Union Rate (Table 1)}

Two weeks after treatment, the bone union rate was significantly higher in the vehicle group than in the PVI group and the EtOH group $(\mathrm{p}<0.001)$ (Table 1). However, the bone union rate was not significantly different between the PVI and $\mathrm{EtOH}$ groups. There was no significant difference among the three groups in the bone union rate 4 weeks after treatment.

\section{Discussion}

In the present study, PVI and EtOH delayed cancellous bone union of the proximal tibia 2 weeks after osteotomy, but there was no significant difference in cancellous bone union 4 weeks after osteotomy. Soaking for 6 minutes at these concentrations of PVI and EtOH significantly delayed early bone healing of the proximal tibia. In regard to the mechanisms of PVI's effects on bone union, it has been reported that PVI is cytotoxic to energy metabolism, cell number, and collagen synthesis of osteoblasts [17]. PVI also causes deleterious effects on alkaline phosphatase production and matrix mineralization in a dose-dependent manner [18]. Recent in vitro studies have demonstrated that a higher concentration of PVI ( $>0.35 \%)$ inhibited osteoblast cellular proliferation, metabolic function, and bone nodule mineralization [6] [19]. These negative effects of PVI on osteoblasts resulted in delayed bone union at the cancellous bone osteotomy site.

In an in vivo study, Husodo et al. reported that $10 \%$ PVI showed a lower percentage of osseous tissue and a higher percentage of fibrous tissue, indicating delayed bone union of the osteotomy site at the femoral shaft of the rat, evaluating mainly cortical bone [20]. Thus, 10\% PVI was selected to evaluate its effect on cancellous bone union at the osteotomy site in the proximal tibia in the present study. However, 1\% PVI did not cause delayed bone union in the previous study [20]. In the present study, $10 \% \mathrm{PVI}$, which is a higher concentration than is used at the surgical site in the clinical situation, was also used. Thus, we should consider the concentration of PVI, since a lower concentration such as $1 \%$ PVI may not adversely affect cancellous bone healing. 
Table 1. Bone union rate at the osteotomy site of the proximal tibia by group.

\begin{tabular}{ccccc}
\hline Bone Union Rate (\%) & Vehicle & PVI & EtOH & ANOVA \\
\hline At 2 weeks & $38.05 \pm 5.20$ & $21.00 \pm 4.64^{a}$ & $19.97 \pm 3.54^{a}$ & $<0.001$ \\
At 4 weeks & $42.46 \pm 22.83$ & $23.10 \pm 7.44$ & $28.24 \pm 6.49$ & 0.091 \\
\hline
\end{tabular}

$n=7$ per group. Values are means \pm standard deviation. PVI: povidone-iodine, EtOH: ethanol. $a$ : $\mathrm{P}<0.001$ vs vehicle group by the Bonferroni test.

On the other hand, EtOH causes no major wound complications such as neurovascular injuries, soft tissue complications, or subsequent fractures [7]. However, it has been reported that EtOH causes tumor necrosis through the degeneration of cellular cytoplasm and proteins, with irreversible vascular thrombosis of small vessels supplying the tumor [21]. EtOH also impairs DNA synthesis and cell proliferation in human osteoblast-like osteosarcoma cells in a dose-dependent manner, although alkaline phosphatase activity remained intact, and accelerated apoptosis was not evident. Thus, it has been reported that the reduction in osteoblasts occurred as a direct effect of $\mathrm{EtOH}$ on proliferative processes of osteoblasts [22]. We have thought that bone union is delayed by thrombosis of intraosseous micro-vessels and the effect on the proliferative processes of osteoblasts. However, there are few previous reports examining whether EtOH inhibits bone healing, and further studies of the effects of EtOH on bone and cartilage formation are needed.

There were several limitations in this study. First, the time points to evaluate the bone union were only 2 and 4 weeks after osteotomy. Earlier time points after osteotomy, such as 3 or 7 days, might be needed to check earlier effects of PVI or EtOH on bone union. Second, the concentrations of PVI and EtOH were determined based on the previous studies, but only a single concentration of PVI or EtOH was used in this study. Future studies are needed to elucidate the negative effects of PVI or EtOH on cancellous bone healing or union under several conditions, such as lower concentrations.

\section{Conclusion}

PVI or EtOH delayed bone union of the cancellous bone osteotomy site of the proximal tibia in the early phase ( 2 weeks), but not at 4 weeks, in rats. Even though the application of PVI or EtOH is useful for preventing surgical site infection or tumor recurrence, respectively, based on the results of the present study, we recommend that PVI or EtOH should be used with some consideration of the adverse effects of PVI or EtOH on local bone at the surgical site.

\section{Acknowledgements}

The authors would like to thank Ms. Matsuzawa and Ms. Kudo for their support in performing the experiments. 


\section{Conflicts of Interest}

The authors declare no conflicts of interest regarding the publication of this paper.

\section{References}

[1] Glotzbecker, B.E., Yolin-Raley, D.S., De Angelo, D.J., Stone, R.M., Soiffer, R.J. and Alyea, E.P. (2013) Impact of Physician Assistants on the Outcomes of Patients with Acute Myelogenous Leukemia Receiving Chemotherapy in an Academic Medical Center. Journal of Oncology Practice, 9, 228-233. https://doi.org/10.1200/JOP.2012.000841

[2] Chundamala, J. and Wrighnt, J.G. (2007) The Efficacy and Risks of Using Povidone-Iodine Irrigation to Prevent Surgical Site Infection: An Evidence-Based Review. Canadian Journal of Surgery, 50, 473-481.

[3] Cheng, M.T., Chang, M.C., Wang, S.T., Yu, W.K., Liu, C.L. and Cen, T.H. (2005) Efficacy of Dilute Betadine Solution Irrigation in the Prevention of Postoperative Infection of Spine Surgery. Spine, 30, 1689-1693.

https://doi.org/10.1097/01.brs.0000171907.60775.85

[4] Viljanto, J. (1980) Disinfection of Surgical Wounds without Inhibition of Normal Wound Healing. The Archives of Surgery, 115, 253-256. https://doi.org/10.1001/archsurg.1980.01380030009003

[5] Ulivieri, S., Toninelli, S., Petrini, C., Giorgio, A. and Oliveri, G. (2011) Prevention of Post-Operative Infections in Spine Surgery by Wound Irrigation with a Solution of Povidone-Iodine and Hydrogen Peroxide. Archives of Orthopaedic and Trauma Surgery, 131, 1203-1206. https://doi.org/10.1007/s00402-011-1262-0

[6] Newton Ede, M.P., Philip, A.M., Philip, A., Richardson, S.M., Mohammad, S. and Jones, S.W. (2016) Povidone-Iodine Has a Profound Effect on in Vitro Osteoblast Proliferation and Metabolic Function and Inhibits Their Ability to Mineralize and Form Bone. Spine, 41, 729-734. https://doi.org/10.1097/BRS.0000000000001332

[7] Szendroi, M. (2004) Giant-Cell Tumour of Bone. The Journal of Bone and Joint Surgery. British Volume, 86, 5-12. https://doi.org/10.1302/0301-620X.86B1.14053

[8] Lin, W.H., Lan, T.Y., Chen, C.Y., Wu, K. and Yang, R.S. (2011) Similar Local Control between Phenol- and Ethanol-Treated Giant Cell Tumors of Bone. Clinical Orthopaedics and Related Research, 469, 3200-3208. https://doi.org/10.1007/s11999-011-1962-3

[9] Oh, J.H., Yoon, P.W., Lee, S.H., Cho, H.S., Kim, W.S. and Kim, H.S. (2006) Surgical Treatment of Giant Cell Tumor of Long Bone with Anhydrous Alcohol Adjuvant. International Orthopaedics, 30, 490-494. https://doi.org/10.1007/s00264-006-0154-3

[10] Nozaka, K., Miyakoshi, N., Kasukawa, Y., Maekawa, S., Noguchi, H. and Shimada, Y. (2007) Intermittent Administration of Human Para-Thyroid Hormone Enhances Bone Formation and Union at the Site of Cancellous Bone Osteotomy in Normal and Ovariectomized Rats. Bone, 42, 90-97. https://doi.org/10.1016/j.bone.2007.08.041

[11] Nithyananth, M., Priscilla, A.J., Boopalan, P.V., Titus, V.T. and Lee, V.N. (2014) Time Required for Effective Action of Phenol against Giant Cell Tumour Cells. Journal of Orthopaedic Surgery, 22, 104-107. https://doi.org/10.1177/230949901402200126

[12] Nozaka, K., Miyakoshi, N., Kasukawa, Y., Maekawa, S., Noguchi, H. and Shimada, Y. (2008) Intermittent Administration of Human Parathyroid Hormone Enhances 
Bone Formation and Union at the Site of Cancellous Bone Osteotomy in Normal and Ovariectimized Rats. Bone, 42, 90-97.

https://doi.org/10.1016/j.bone.2007.08.041

[13] Tsuchie, H., Miyakoshi, N., Kasukawa, Y., Aonuma, H. and Shimada, Y. (2011) The Effects of Circumferential Wiring on Cancellous Bone Healing after Osteotomy in Ovariectomized Rats. Akita Journal of Medicine, 38, 21-26.

[14] Nakajima, A., Nakajima, F., Shimizu, S., Ogasawara, A., Wanaka, A., Moriya, H., Einhorn, T.A. and Yamazaki, M. (2001) Spatial and Temporal Gene Expression for Fibroblast Growth Factor Type I Receptor (FGFR1) during Fracture Healing in the Rat. Bone, 29, 458-466. https://doi.org/10.1016/S8756-3282(01)00604-4

[15] Kawano, T., Miyakoshi, N., Kasukawa, Y., Hongo, M., Tsuchie, H., Sato, C., Fujii, M., Suzuki, M., Akagawa, M., Ono, Y., Yuasa, Y., Nagahata, I. and Shimada, Y. (2017) Effects of Combined Therapy of Alendronate and Low-Intensity Pulsed Ultrasound on Metaphyseal Bone Repair after Osteotomy in the Proximal Tibia of Glucocorticoid-Induced Osteopenia Rats. Osteoporosis and Sarcopenia, 3, 185-191. https://doi.org/10.1016/j.afos.2017.11.001

[16] Kanda, Y. (2013) Investigation of the Freely-Available Easy-to-Use Software "EZR" (Easy R) for Medical Statistics. Bone Marrow Transplant, 48, 452-458. https://doi.org/10.1038/bmt.2012.244

[17] Kaysinger, K.K., Nicholson, N.C., Ramp. W.K. and Kellam, J.F. (1995) Toxic Effects of Wound Irrigation Solutions on Cultured Tibiae and Osteoblasts. Journal of Orthopaedic Trauma, 9, 303-311. https://doi.org/10.1097/00005131-199509040-00006

[18] Cabral, C.T. and Fernandes, M.H. (2007) In Vitro Comparison of Chlorhexidine and Povidone-Iodine on the Long-Term Proliferation and Functional Activity of Human Alveolar Bone Cells. Clinical Oral Investigations, 11, 155-164. https://doi.org/10.1007/s00784-006-0094-8

[19] Liu, J.X., Werner, J.A., Buza, J.A., Kirsch, T., Zuckerman, J.D. and Virk, M.S. (2017) Povidone-Iodine Solutions Inhibit Cell Migration and Survival of Osteoblasts, Fibroblasts, and Myoblasts. Spine, 42, 1752-1756. https://doi.org/10.1097/BRS.0000000000002224

[20] Husodo, K., Kamal, A.F. and Yusuf, A.A. (2016) Effect of Povidone Iodine and Hydrogen Peroxide on Fracture Healing: A Histomorphometric Study on Rats. Journal of Orthopaedic Surgery, 24, 245-249. https://doi.org/10.1177/1602400224

[21] Shiina, S., Tagawa, K., Unuma, T., Takanashi, R., Yoshiura, K., Komatsu, Y., Hata, Y., Niwa, Y., Shiratori, Y. and Terano, A. (1991) Percutaneous Ethanol Injection Therapy for Hepatocellular Carcinoma. A Histopathologic Study. Cancer, 68, 1524-1530. https://doi.org/10.1002/1097-0142(19911001)68:7<1524::AID-CNCR2820680711>3. $\underline{0 . \mathrm{CO} ; 2-\mathrm{O}}$

[22] Klein, R.F., Fausti, K.A. and Carlos, A.S. (1996) Ethanol Inihibits Human Osteoblastic Cell Proliferation. Alcoholism: Clinical and Experimental Research, 20, 572-578. https://doi.org/10.1111/j.1530-0277.1996.tb01095.x 